\title{
Synthesis of fused heterocycles utilizing the heterocyclic enamine protocol
}

\author{
Yong Liu, Chu-Yi Yu and Mei-Xiang Wang* \\ Laboratory of Chemical Biology, Center for Molecular Science, Institute of Chemistry, Chinese \\ Academy of Sciences, Beijing 100080, China \\ E-mail: mxwang@mail.iccas.ac.cn
}

Dedicated to Professor Zhi-Tang Huang on His $75^{\text {th }}$ Birthday

(received 21 Feb 03; accepted 11 May 03; published on the web 17 May 03)

\begin{abstract}
Heterocyclic enamines 1 underwent regioselective $C$-benzylation and $C$-benzoylation with $o$ bromobenzyl bromide and $o$-halobenzoyl chloride to yield the corresponding $C$-benzylated and $C$-benzoylated heterocyclic enamines $\mathbf{2}$ and $\mathbf{8}$, respectively. Subsequent intramolecular substitution reactions of $\mathbf{2}$ and of $\mathbf{8}$ led to, respectively, the fused 1,4-dihydroquinolines $\mathbf{3}$ and quinolin-4-ones $\mathbf{9}$. The ring size effect of the heterocyclic enamines on the reactivity of both the enaminic carbon and the secondary amine was observed.
\end{abstract}

Keywords: Heterocyclic enamines, benzylation, benzoylation, fused 1,4-dihydroquinolines, fused quinolin-4-ones

\section{Introduction}

Heterocyclic secondary enamines 1 (Figure 1), also known as exo-cyclic enaminoesters, enaminones, and enamino nitriles and the like, are versatile intermediates for the synthesis of natural products and heterocyclic compounds. The chemistry of heterocyclic enamines was pioneered by Eschenmoser and his coworkers ${ }^{1}$ in the 1960 s during their synthetic studies of corrin. They used the reaction of an enamino nitrile with iminoether as one of the key steps in the construction of the corrin ring system. Since the late 1970s, heterocyclic enamines have been investigated by Kishi, ${ }^{2}$ Danishefsky, ${ }^{3-5}$ Rapoport $^{6}$ and others in the synthesis of saxitoxin, camptothecin, mitomycins and alkaloids. ${ }^{7-12}$ And recently, exo-cyclic enaminoesters have been employed in the the synthesis of carbacephems ${ }^{13}$, a new class of $\beta$-lactam antibiotics. 


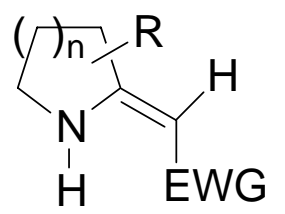

$$
\begin{aligned}
& \text { EWG }=\mathrm{CO}_{2} \mathrm{R}^{\prime}, \text { COR', CN } \\
& \mathrm{n}=1,2,3 \\
& \mathrm{R}=\text { alkyl, aryl, alkoxy }
\end{aligned}
$$

1

Figure 1. Structure of heterocyclic enamines

One of the most noticeable features of heterocyclic secondary enamines $\mathbf{1}$ is their ambident bisnucleophilicity; nucleophilic reaction can occur at the enaminic carbon and/or the secondary amino nitrogen. This has been exemplified ${ }^{14,15}$ by the acylation reaction using simple carboxylic acid chlorides, which gave rise to $C$ - and/or N-acylated products depending upon both the heterocyclic structure of enamines and the structure of carboxylic acids. Much attention has been given to the annulation reactions between heterocyclic enamines and $\alpha, \beta$-unsaturated compounds in the past decades ${ }^{16,17}$ and a systematic investigation of the reaction of exo-cyclic enamino esters with electrophilic alkynes has been reported. ${ }^{18}$ Very recently, the unexpected diversity of reaction patterns of heterocyclic enamines when treated with aliphatic and aromatic dicarboxylic acid chlorides has been discovered. ${ }^{19}$ Our interest ${ }^{18,19}$ in exploiting the intriguing reactivity of heterocyclic enamines and their application in the synthesis of natural products such as polyhydroxylated pyrrolizidine ${ }^{20}$ and indolizidine ${ }^{21}$ alkaloids and their analogs led us to the current study. We wish to report herein the convenient synthesis of fused N-heterocycles from selective C-benzylation and benzoylation of heterocyclic enamines followed by intramolecular cyclization.

\section{Results and Discussion}

We first tested the reaction of heterocyclic enamines $1 \mathbf{1 a - \mathbf { c } ^ { 2 2 }}$ with $o$-bromobenzyl bromide, and found an interesting ring size effect on the reaction. For example, seven-membered heterocyclic enamine 1c reacted with o-bromobenzyl bromide in refluxing acetonitrile to provide 2c in 56\% yield, while no reaction was observed for five- and six-membered enamine analogs $\mathbf{1 a}$ and $\mathbf{1 b}$ under the same neutral conditions. Only in the presence of a strong base such as $\mathrm{NaH}$, did the reaction proceed smoothly to give C-benzylated product $\mathbf{2 a}$ or $\mathbf{2 b}$ in moderate yields (Scheme 1). Although a mixture of uncharacterizable by-products was formed, no N-benzylated compound was observed and isolated. It is interesting to note that the reaction of heterocyclic enamines $\mathbf{1}$ proceeded in a totally different pathway from its analogous heterocyclic enediamines or heterocyclic ketene aminals, powerful and versatile synthetic intermediates developed by Professor Zhi-Tang Huang, which afforded exclusively N-benzylated products under identical basic conditions $^{23}$ (see also the Tribute). The C-benzylated heterocyclic enamine structure 2 and $Z$-configuration of the double bond was evidenced by the observation of disappearance of the vinyl proton signal and of the downfield shift of $\mathrm{NH}$ proton signal, due to the strong 
intramolecular hydrogen bonding to the ester carbonyl, in ${ }^{1} \mathrm{H}$ NMR spectrum. Intramolecular cyclization of $\mathbf{2 a}$ or $\mathbf{2} \mathbf{b}$ via Pd-catalyzed coupling reaction of secondary enamine nitrogen with bromobenzene moiety furnished the fused heterocyclic compounds $\mathbf{3 a}$ or $\mathbf{3 b}$. The same reaction of 2c, however, gave a mixture of decomposed compounds and no $\mathbf{3 c}$ was obtained. The isomerization of enaminoester double bond occurred during the cyclization reaction. The lower chemical conversion was most probably due to the weaker nucleophilicity of secondary amine resulted from the formation of conjugative enaminoester system.

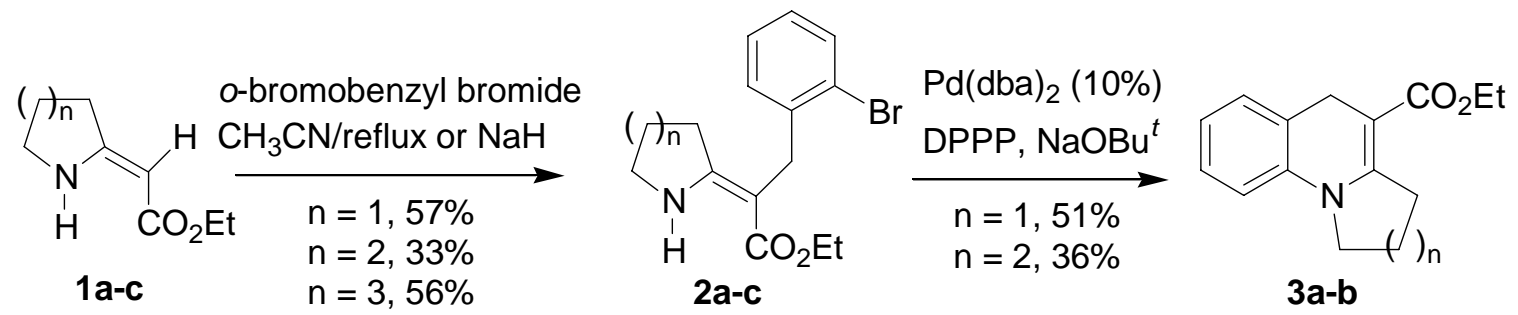

Scheme 1. Synthesis of fused 1,4-dihydroquinoline derivatives.

Michael et al. ${ }^{24}$ once reported that intramolecular Heck reaction of N-arylated heterocyclic enamine 4a yielded indole derivative $5 \mathbf{a}^{25}$ We concurred with their finding and extended this method to the preparation of piperidino[1,2-b]indole product $\mathbf{5 b}$ (Scheme 2). Encouraged by these results, the synthesis of pyrrolo[1,2-b]quinolin-4-one $\mathbf{9}^{26}$ through enaminic cyclization of $\mathrm{N}$-(2-ethyoxycarbonyl)phenyl heterocyclic enamine $\mathbf{6}$ was then attempted. However, no reaction was effected under both neutral and basic conditions, probably because of the lower enaminic reactivity of $\mathbf{6}$ towards ester group. In the presence of polyphosphoric acid (PPA) or $p$ toluenesulfonic acid ( $\mathrm{TsOH})$, enamine 6 did undergo a smooth reaction to yield, instead of the desired fused heterocycle, the ring-opening product 7 . The proposed reaction pathway, which depicted in Scheme 3, comprised the hydration of enamine double bond followed by ring-chain tautomerization and hydrolytic decarboxylation steps.<smiles>CCOC(=O)/C=C1/CCCN1c1ccccc1Br</smiles>

4a-b
$\mathrm{Pd}(\mathrm{OAC})_{2}, \mathrm{PPh}_{3}$

$\mathrm{NEt}_{3} / \mathrm{CH}_{3} \mathrm{CN}$

$\mathrm{n}=1,90 \%$

$\mathrm{n}=2,51 \%$

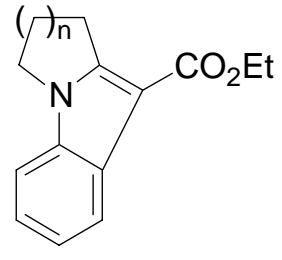

5a-b

Scheme 2. Synthesis of fused indole derivatives 5. 
<smiles>CCOC(=O)/C=C1/CCCN1c1ccccc1C(=O)OCC</smiles>

6

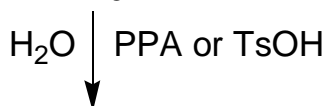

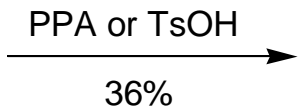

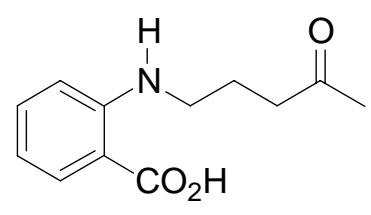

7<smiles>CC(=O)O</smiles>

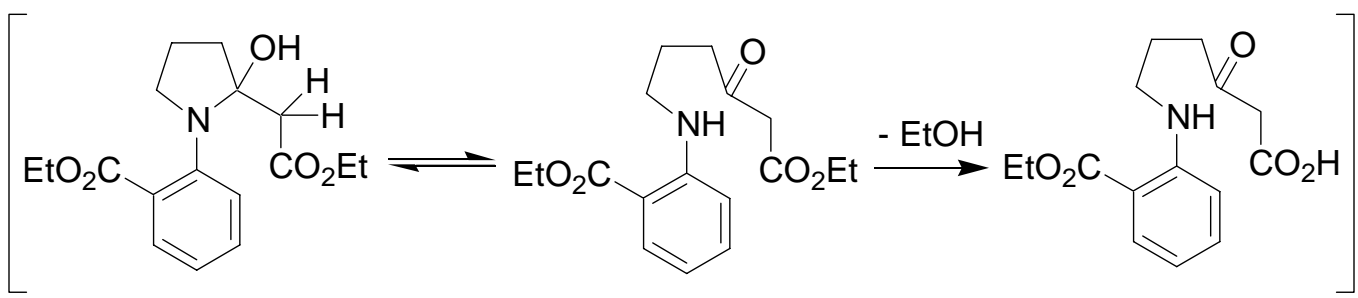

Scheme 3. Hydrolysis of heterocyclic enamine 6.

To prepare fused quinolin-4-one derivatives 9 we then took an alternative strategy based on annulation reaction of heterocyclic enamines 1 with $o$-halobenzoic acid chloride. In the presence of pyridine, enamine 1a underwent a regiospecific $C$-benzoylation with $o$-chlorobezoyl chloride to give 8a. No $N$-benzoylated product was observed. Subsequent cyclization reaction was examined by the treatment with $\mathrm{NaH}$ in THF at $60^{\circ} \mathrm{C}$. However, no reaction was observed, even at higher temperature such as in refluxing DMF or 1,4-dioxane. When $\mathrm{Cl}$ was replaced by $\mathrm{Br}$ using $o$-bromobezoyl chloride as the starting benzoylation reagent, the resulting 8a' cyclized readily under basic conditions to afford pyrrozolo[1,2-a]quinolone 9a in $40 \%$ yield. Around $12 \%$ yield of enamine 1a was also obtained, which probably resulted from debenzoylation reaction of 8a'. In the case of the six- and the seven-membered enamines, however, the $C$-benzoylated products $\mathbf{8 b}$ and $\mathbf{8 c}(\mathrm{X}=\mathrm{Cl})$, which were not purified, underwent effective cyclization reaction to yield fused quinolin-4-ones $\mathbf{9 b}$ and $\mathbf{9 c}$, respectively. The ready conversion of $\mathbf{8 b}, \mathbf{c}$ to tricyclic compounds $\mathbf{9 b , c}$ was most likely due to the combined effect of higher nucleophilic reactivity of the secondary amino group of the six- and seven-membered heterocyclic enamine species and the less ring strain of the fused heterocyclic ring systems of $\mathbf{9 b}$ and $\mathbf{9 c}$

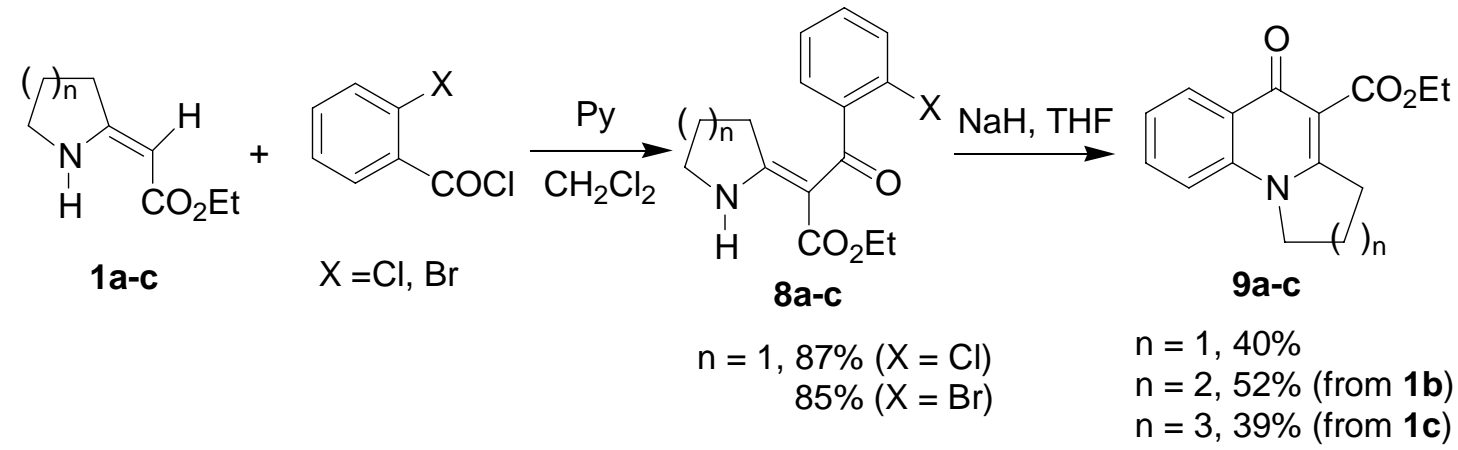

Scheme 4. Synthesis of fused quinolin-4-one derivatives 9 . 
In conclusion, we have shown that heterocyclic enamines $\mathbf{1}$ are versatile bis-nucleophiles able to react with bis-electrophiles such as o-bromobenzyl bromide and $o$-halobenzoic acid chloride, and their synthetic application has been demonstrated by the preparation of fused 1,4dihydroquinoline and quinolin-4-one derivatives. It would be highly interesting and challenging to prepare chiral (poly)hydroxylated fused $N$-heterocycles, that are analogs or precursors of (poly)hydroxylated alkaloids, starting from chiral (poly)hydroxylated heterocyclic enamines.

\section{Experimental Section}

General Procedures. Melting points are uncorrected. ${ }^{1} \mathrm{H}$ NMR and ${ }^{13} \mathrm{C}$ NMR spectra were obtained on a Bruker 300 spectrometer. IR spectra were obtained on a HITACHI-260-50 spectrometer as liquid films or $\mathrm{KBr}$ discs. Mass spectra were measured on AEI MS-50 (EI) and KYKY-ZHT-5 (FAB) mass spectrometers. Exact mass spectra were obtained on a Bruker APEX-2 mass spectrometer. Elemental analyses were performed at the Analytical Laboratory of the Institute. All chemicals were dried or purified according to standard procedures prior to use.

\section{Synthesis of $C$-benzylated heterocyclic enamines 2a-c}

(Z)-Ethyl 3-(2-bromophenyl)-2-pyrrolidin-2-ylidene propionate (2a). To a solution of 1a in $\mathrm{DMF}$ at $0{ }^{\circ} \mathrm{C}$ was added $\mathrm{NaH}$ (1.2 eq.) in one portion. After stirring for $30 \mathrm{~min}$ at $0{ }^{\circ} \mathrm{C}, \mathrm{o}^{-}$ bromobenzyl bromide (1.2 eq.) was added. The mixture was heated at $80{ }^{\circ} \mathrm{C}$ for $24 \mathrm{~h}$ and then cooled to room temperature. The reaction was quenched by adding saturated aqueous $\mathrm{NH}_{4} \mathrm{Cl}$ solution and extracted with ethyl acetate. The extracts combined were washed with $\mathrm{H}_{2} \mathrm{O}$, brine and dried over with $\mathrm{Na}_{2} \mathrm{SO}_{4}$. Column chromatography afforded 2a as white solid in 57\% yield: mp 75-76 ${ }^{\circ} \mathrm{C}$; IR (KBr) 3371, 1657, $1588 \mathrm{~cm}^{-1}$; ${ }^{1} \mathrm{H}$ NMR (300 MHz, $\left.\mathrm{CDCl}_{3}\right) \delta 8.37$ (s, br., $1 \mathrm{H}$, $\mathrm{NH}$ ), 7.52 (d, J 7.8 Hz, 1H, ArH), 7.20-7.00 (m, 3H, ArH), 4.13 (q, J 7.1 Hz, 2H, OCH $), 3.63$ (s, 2H, $\mathrm{CH}_{2}$ ), 3.57 (t, J $7.0 \mathrm{~Hz}, 2 \mathrm{H}, \mathrm{CH}_{2}$ ), 2.52 (t, $J .7 \mathrm{~Hz}, 2 \mathrm{H}, \mathrm{CH}_{2}$ ), 1.99 (quin, $J .3 \mathrm{~Hz}, 2 \mathrm{H}$, $\mathrm{CH}_{2}$ ), $1.16\left(\mathrm{t}, J 7.0 \mathrm{~Hz}, 3 \mathrm{H}, \mathrm{CH}_{3}\right) ;{ }^{13} \mathrm{C} \mathrm{NMR}\left(75 \mathrm{MHz}, \mathrm{CDCl}_{3}\right) \delta$ 170.9, 166.4, 141.9, 132.5, 129.1, 127.5, 127.3, 125.0, 86.1, 59.1, 47.7, 34.1, 31.4, 22.3, 15.0; MS (EI) m/z 325 (30), 323 ( $\mathrm{M}^{+}-1$, 30\%), 296 (17), 294 (18), 278 (18), 252 (40), 250 (48), 244 (100). Anal. Calcd for $\mathrm{C}_{15} \mathrm{H}_{18} \mathrm{NO}_{2} \mathrm{Br}$ : C, 55.57; H, 5.60; N, 4.32. Found: C, 55.77; H, 5.66; N, 4.14.

(Z)-Ethyl-3-(2-bromophenyl)-2-piperidin-2-ylidene propionate (2b). Following the same procedure as for $\mathbf{2 a}$, product $\mathbf{2 b}$ was obtained in $33 \%$ yield as white solid: $\mathrm{mp} 52-53{ }^{\circ} \mathrm{C}$; IR (KBr) 3150, 1636, $1601 \mathrm{~cm}^{-1}$; ${ }^{1} \mathrm{H}$ NMR (300 MHz, $\mathrm{CDCl}_{3}$ ) $\delta 9.78$ (s, br., 1H, NH), 7.44 (d, J 7.8

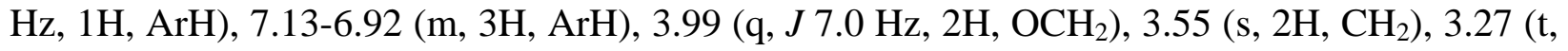
$\left.J 5.8 \mathrm{~Hz}, 2 \mathrm{H}, \mathrm{CH}_{2}\right), 2.18$ (t, J $\left.6.4 \mathrm{~Hz}, 2 \mathrm{H}, \mathrm{CH}_{2}\right), 1.64-1.54\left(\mathrm{~m}, 4 \mathrm{H}, \mathrm{CH}_{2} \mathrm{CH}_{2}\right), 1.07$ (t, J 7.1 Hz, $\left.3 \mathrm{H}, \mathrm{CH}_{3}\right) ;{ }^{13} \mathrm{C} \mathrm{NMR}\left(75 \mathrm{MHz}, \mathrm{CDCl}_{3}\right) \delta 170.8,161.7,141.4,131.9,128.3,127.0,126.7,124.5$, 86.7, 58.4, 41.3, 32.0, 25.8, 22.0, 19.8, 14.4; MS (EI) m/z 339 (26), 337 (M+1, 28\%), 310 (18), 308 (18), 292 (14), 266 (54), 264 (61) 258 (100). Anal. Calcd for $\mathrm{C}_{16} \mathrm{H}_{20} \mathrm{NO}_{2} \mathrm{Br}$ : 337.0671. Found: 337.0674. 
(Z)-Ethyl 2-azepan-2-ylidene-3-(2-bromophenyl)propionate (2c). A mixture of 1c and $o$ bromobenzyl bromide (1.2 eq.) in acetonitrile was refluxed 24h. After removal of solvent, the residue was subjected to column chromatography to afford 2c in 56\% yield as oil: IR (KBr) 3272, 1638, $1589 \mathrm{~cm}^{-1}$; ${ }^{1} \mathrm{H}$ NMR (300 MHz, $\mathrm{CDCl}_{3}$ ) $\delta 9.84$ (s, br., 1H, NH), 7.49 (d, J 7.8 Hz, 1H, ArH), (7.20-6.97 (m, 3H, ArH), 4.06 (q, J 6.9 Hz, 2H, $\mathrm{OCH}_{2}$ ), 3.70 (s, 2H, $\mathrm{CH}_{2}$ ), 3.36-3.31 (m, 2H, $\mathrm{CH}_{2}$ ), 2.32-2.28 (m, 2H, $\left.\mathrm{CH}_{2}\right), 1.64-1.15$ (m, 4H, $\left.\mathrm{CH}_{2} \mathrm{CH}_{2}\right), 1.48-1.1 .42$ (m, $2 \mathrm{H}, \mathrm{CH}_{2}$ ), $1.14\left(\mathrm{t}, J 7.0 \mathrm{~Hz}, 3 \mathrm{H}, \mathrm{CH}_{3}\right) ;{ }^{13} \mathrm{C} \mathrm{NMR}\left(75 \mathrm{MHz} \mathrm{CDCl}_{3}\right) \delta 171.5,168.4,142.5,132.4,129.5$, 127.4, 127.3, 124.7, 87.8, 59.1, 44.3, 33.4, 30.7, 30.4, 29.4, 25.6, 14.9; MS (EI) m/z 353 (57), 351 ( $\left.\mathrm{M}^{+}-1,58 \%\right), 324$ (32), 322 (32), 308 (18), 306 (19), 280 (68), 278 (77), 272 (100). Anal. Calcd for $\mathrm{C}_{17} \mathrm{H}_{22} \mathrm{NO}_{2} \mathrm{Br}$ : 351.0828. Found: 351.0829.

\section{General procedure for the synthesis of fused 1,4-dihydroquinolines (3)}

To a solution of 2 in toluene was added Pd(dba) $)_{2}(10 \% \mathrm{~mol})$, DPPP $(10 \% \mathrm{~mol})$ and $\mathrm{NaOBu}^{\mathrm{t}}(1.2$ eq.). The mixture was heated at $110^{\circ} \mathrm{C}$ for $24 \mathrm{~h}$ under argon. After filtration of the catalyst, the filtrate was diluted with ether, washed with $\mathrm{H}_{2} \mathrm{O}$, brine and dried over $\mathrm{Na}_{2} \mathrm{SO}_{4}$. After removal of the solvents, the residue was subjected to column chromatography to yield 3 .

Ethyl 1,2,3,5-tetrahydropyrrolo[1,2-a]quinoline-4-carboxylate (3a). 51\%; oil; IR (KBr) 1665, $1634 \mathrm{~cm}^{-1}$; ${ }^{1} \mathrm{H}$ NMR (300 MHz, $\left.\mathrm{CDCl}_{3}\right) \delta 7.11$ (t, $\left.J 8.4 \mathrm{~Hz}, 1 \mathrm{H}, \mathrm{ArH}\right), 7.10$ (d, $J 8.3 \mathrm{~Hz}, 1 \mathrm{H}$, ArH), 6.94 (t, J 7.2 Hz, 1H, ArH), 6.65 (d, J 7.9 Hz, 1H, ArH), 4.18 (q, J 7.1 Hz, 2H, OCH $)$, 3.79 (s, 2H, $\mathrm{CH}_{2}$ ), 3.59 (t, $J 7.0 \mathrm{~Hz}, 2 \mathrm{H}, \mathrm{CH}_{2}$ ), 3.14 (t, $J .7 \mathrm{~Hz}, 2 \mathrm{H}, \mathrm{CH}_{2}$ ), 2.12 (quin, $J .3 \mathrm{~Hz}$, 2H, $\mathrm{CH}_{2}$ ), 1.30 (t, J 7.1 Hz, 3H, $\mathrm{CH}_{3}$ ); ${ }^{13} \mathrm{C} \mathrm{NMR} \mathrm{(75} \mathrm{MHz,} \mathrm{CDCl}_{3}$ ) $\delta 168.5,155.9,138.9,129.2$, 127.3, 124.3, 123.2, 112.9, 90.9, 59.6, 48.7, 32.4, 30.1, 28.1, 22.0, 15.1; MS (EI) m/z $243\left(\mathrm{M}^{+}\right.$, 21\%), 242 (27), 214 (100). Anal. Calcd for $\mathrm{C}_{15} \mathrm{H}_{16} \mathrm{NO}_{2}$ : 242.1175. Found: 242.1174.

Ethyl 2,3,4,6-tetrahydro-1H-pyrido[1,2-a]quinoline-5-carboxylate (3b). 36\%; oil; IR (KBr) 1683, $1604 \mathrm{~cm}^{-1} ;{ }^{1} \mathrm{H}$ NMR (300 MHz, $\mathrm{CDCl}_{3}$ ) $\delta 7.16$ (t, J 8.0 Hz, 1H, ArH), 7.10 (d, J 7.2 Hz, 1H, ArH), 6.99 (t, J 7.3 Hz, 1H, ArH), 6.88 (d, J $8.2 \mathrm{~Hz}, 1 \mathrm{H}, \mathrm{ArH}), 4.18$ (q, J $7.1 \mathrm{~Hz}, 2 \mathrm{H}$, $\mathrm{OCH}_{2}$ ), 3.66 (s, 2H, CH ), 3.63 (t, $J 5.9 \mathrm{~Hz}, 2 \mathrm{H}, \mathrm{CH}_{2}$ ), 3.22 (t, $J 7.0 \mathrm{~Hz}, 2 \mathrm{H}, \mathrm{CH}_{2}$ ), 1.93 (quin, $J$ $6.3 \mathrm{~Hz}, 2 \mathrm{H}, \mathrm{CH}_{2}$ ), 1.75 (quin, $J 6.8 \mathrm{~Hz}, 2 \mathrm{H}, \mathrm{CH}_{2}$ ), 1.31 (t, $\left.J 7.1 \mathrm{~Hz}, 3 \mathrm{H}, \mathrm{CH}_{3}\right) ;{ }^{13} \mathrm{C}$ NMR $(75$ $\left.\mathrm{MHz} \mathrm{CDCl}_{3}\right) \delta 167.9,153.6,141.4,128.1,126.5,125.0,122.8,112.4$, 94.6, 59.3, 45.0, 28.0, 26.5, 22.6, 19.2, 14.6. Anal. Calcd for $\mathrm{C}_{16} \mathrm{H}_{18} \mathrm{NO}_{2}$ : 256.1332. Found: 256.1329.

\section{General procedure for the synthesis of fused indole derivatives (5)}

To a solution of 4 in $\mathrm{CH}_{3} \mathrm{CN}$ was added $\mathrm{Et}_{3} \mathrm{~N}$ (2 eq.), $\mathrm{Pd}(\mathrm{OAc})_{2}$ (1 eq.) and $\mathrm{PPh}_{3}$ (2 eq.). The mixture was heated at $80{ }^{\circ} \mathrm{C}$ for $24 \mathrm{~h}$ under argon. After filtration of the catalyst, the filtrate was subjected to column chromatography to give 5 .

Ethyl 2,3-dihydro-1H-pyrrolo[1,2-a]indole-9-carboxylate 5a. 90\% as white solid; mp 94-95 ${ }^{\circ} \mathrm{C}$ (lit. 96-98.5 ${ }^{\circ} \mathrm{C}^{25 \mathrm{a}}, 95.6-96{ }^{\circ} \mathrm{C}^{25 \mathrm{~b}}$ ); ${ }^{1} \mathrm{H} \mathrm{NMR}\left(300 \mathrm{MHz}, \mathrm{CDCl}_{3}\right) \delta 8.04$ (d, J 7.9 Hz, 1H, ArH), 7.19-7.13 (m, 3H, ArH), 4.30 (q, J 7.1 Hz, 2H, CH ), 4.05 (t, J 7.2 Hz, 2H, CH 2 ), 3.23 (t, J 7.6 $\mathrm{Hz}, 2 \mathrm{H}, \mathrm{CH}_{2}$ ), 2.59 (quin, J 7.3 Hz, 2H, $\mathrm{CH}_{2}$ ), 1.35 (t, $\left.J .1 \mathrm{~Hz}, 3 \mathrm{H}, \mathrm{CH}_{3}\right) ;{ }^{13} \mathrm{C} \mathrm{NMR}(75 \mathrm{MHz}$, $\left.\mathrm{CDCl}_{3}\right) \delta 165.6,152.8,132.6,130.9,121.6,121.5,121.4,109.8,99.3,59.3,44.4,26.6,26.1$, 14.7; MS (EI) m/z 230 (12), 229 (M+, 42\%), 200 (27), 184 (54), 58 (100).

Ethyl-6,7,8,9-tetrahydropyrido[1,2-a]indole-10-carboxylate 5b. 51\%; IR (KBr) $1684 \mathrm{~cm}^{-1}$; ${ }^{1} \mathrm{H}$ NMR (300 MHz, $\left.\mathrm{CDCl}_{3}\right) \delta 8.14$ (d, J 7.4 Hz, 1H, ArH), 7.26-7.20 (m, 3H, ArH), 4.39 (q, J 7.0 
$\mathrm{Hz}, 2 \mathrm{H}, \mathrm{OCH}_{2}$ ), 4.07 (t, $J 6.0 \mathrm{~Hz}, 2 \mathrm{H}, \mathrm{CH}_{2}$ ), 3.34 (t, J $6.2 \mathrm{~Hz}, 2 \mathrm{H}, \mathrm{CH}_{2}$ ), 2.11-2.08 (m, 2H, $\mathrm{CH}_{2}$ ), 1.98-1.92 (m, 2H, $\mathrm{CH}_{2}$ ), 1.45 (t, $\left.J 7.0 \mathrm{~Hz}, 3 \mathrm{H}, \mathrm{CH}_{3}\right) ;{ }^{13} \mathrm{C} \mathrm{NMR}\left(75 \mathrm{MHz}, \mathrm{CDCl}_{3}\right.$ ) $\delta$ 166.4, 146.2, 136.3, 127.0, 122.3, 122.0, 121.5, 109.2, 102.9, 59.6, 42.8, 25.0, 22.9, 20.4, 15.0; MS (EI) $\mathrm{m} / \mathrm{z}$ 244 (25), 243 ( $\left.\mathrm{M}^{+}, 46 \%\right), 214$ (82), 197 (61), 168 (100). Anal. Calcd for $\mathrm{C}_{15} \mathrm{H}_{17} \mathrm{NO}_{2}$ : 243.1254. Found: 243.1254.

Attempted synthesis of fused quinolin-4-one derivative 8a from the reaction of heterocyclic enamine (6)

A mixture of $\mathbf{6}$ and TsOH (2 eq.) in ethanol or acetonitrile was refluxed for $2 \mathrm{~h}$. After removal of solvent, the residue was neutralized with saturated aqueous $\mathrm{Na}_{2} \mathrm{CO}_{3}$ solution and extracted with EtOAc. The organic layer was washed with brine and $\mathrm{H}_{2} \mathrm{O}$ and then dried over with $\mathrm{Na}_{2} \mathrm{SO}_{4}$. After column chromatography, ethyl 2-(4-oxopentylamino)benzoate 7 was obtained in 36\% yield as a colorless oil; IR (KBr) 3362, 1716, $1680 \mathrm{~cm}^{-1} ;{ }^{1} \mathrm{H}$ NMR (300 MHz, $\left.\mathrm{CDCl}_{3}\right) \delta 7.96$ (d, J 7.9 Hz, 1H, ArH), 7.77 (s, br., 1H, NH), 7.39 (t, J 7.8 Hz, 1H, ArH), 6.74 (d, J 8.5 Hz, 1H, ArH), 6.62 (t, $J 7.5 \mathrm{~Hz}, 1 \mathrm{H}, \mathrm{ArH}), 4.35$ (q, J 7.1 Hz, 2H, OCH ), 3.27 (q, $J 5.9 \mathrm{~Hz}, 2 \mathrm{H}, \mathrm{CH}_{2}$ ), 2.63 (t, $J$ $7.1 \mathrm{~Hz}, 2 \mathrm{H}, \mathrm{CH}_{2}$ ), 2.20 (s, 3H, $\mathrm{CH}_{3}$ ), 1.99 (quin, J 7.0 Hz, 2H, $\mathrm{CH}_{2}$ ), 1.42 (t, J 7.0 Hz, 3H, $\mathrm{CH}_{3}$ ); ${ }^{13} \mathrm{C}$ NMR $\left(75 \mathrm{MHz}, \mathrm{CDCl}_{3}\right.$ ) $\delta$ 207.8, 168.6, 151.1, 134.5, 131.6, 114.4, 111.1, 110.0, 60.1, 41.9, 40.7, 29.9, 23.0, 14.3; MS (EI) m/z 249 (M+, 15\%), 191 (29), 178 (20), 145 (17), 132 (100). Anal. Calcd for $\mathrm{C}_{14} \mathrm{H}_{19} \mathrm{NO}_{3}$ : 249.1359. Found: 249.1353 .

\section{Synthesis of fused quinolin-4-one derivatives (9)}

\section{A. Benzoylation reaction of heterocyclic enamines 1}

(Z)-Ethyl 3-(2-chlorophenyl)-3-oxo-2-(pyrrolidin-2-ylidene)propionate (8a). To a mixture of 1a and pyridine (2 eq.) in $\mathrm{CH}_{2} \mathrm{Cl}_{2}$ at $0^{\circ} \mathrm{C}$ was added $o$-cholorobenzoyl chloride. After stirring for $30 \mathrm{~min}$ at room temperature, the mixture was refluxed for $6 \mathrm{~h}$, and then cooled and diluted with $\mathrm{CH}_{2} \mathrm{Cl}_{2}$. The organic solution was washed with saturated sodium bicarbonate solution, $\mathrm{H}_{2} \mathrm{O}$, citric acid (10\%) and brine, and then dried over with anhydrous $\mathrm{Na}_{2} \mathrm{SO}_{4}$. Column chromatography of the residue gave 8a (87\%) as white solid: mp 124-125 ${ }^{\circ} \mathrm{C}$; IR (KBr) 3137, 1699, 1669, $1615 \mathrm{~cm}^{-1}$; ${ }^{1} \mathrm{H}$ NMR (300 MHz, $\mathrm{CDCl}_{3}$ ) $\delta 7.60$ (d, J 8.3 Hz, 1H, ArH), 7.43-7.29 (m, 3H, ArH), 6.84 (s, br., 1H, NH), 4.14 (q, J $7.1 \mathrm{~Hz}, 2 \mathrm{H}, \mathrm{OCH}_{2}$ ), 3.53 (s, br., 2H, $\mathrm{CH}_{2}$ ), 3.25 (t, $J$ $7.6 \mathrm{~Hz}, 2 \mathrm{H}, \mathrm{CH}_{2}$ ), 1.91 (quin, J $7.3 \mathrm{~Hz}, 2 \mathrm{H}, \mathrm{CH}_{2}$ ), 1.26 (t, J 7.1 Hz, 3H, $\mathrm{CH}_{3}$ ); ${ }^{13} \mathrm{C}$ NMR $(75$ $\left.\mathrm{MHz}_{\mathrm{CDCl}}\right) \delta$ 168.4, 167.4, 156.0, 130.7, 129.8, 129.4, 127.4, 127.1, 100.6, 59.4, 51.0, 31.7, 21.9, 14.3; MS (EI) m/z $258\left(\mathrm{M}^{+}-35\right.$, 33\%), 141 (32), 139 (100). Anal. Calcd for $\mathrm{C}_{15} \mathrm{H}_{16} \mathrm{NO}_{3} \mathrm{Cl}$ : C, 61.33; H, 5.49; N, 4.77. Found: C, 61.35; H, 5.52; N, 4.66.

(Z)-Ethyl 3-(2-bromophenyl)-3-oxo-2-(pyrrolidin-2-ylidene)propionate (8a'). Following the same procedure as for $\mathbf{8 a}$, the reaction of $\mathbf{1 a}$ with $o$-bromobenzoyl chloride produced $\mathbf{8 a}$ (85\%) as white solid: mp 119-120 ${ }^{\circ} \mathrm{C}$; IR (KBr) 3136, 1698, 1669, $1615 \mathrm{~cm}^{-1}$; ${ }^{1} \mathrm{H}$ NMR (300 MHz, $\left.\mathrm{CDCl}_{3}\right) \delta 7.60$ (d, J 8.3 Hz, 1H, ArH), 7.43-7.29 (m, 3H, ArH), 6.95 (s, br., 1H, NH), 4.15 (q, J $7.0 \mathrm{~Hz}, 2 \mathrm{H}, \mathrm{OCH}_{2}$ ), 3.58-3.47 (m, 2H, $\mathrm{CH}_{2}$ ), 3.29 (t, $J 7.8 \mathrm{~Hz}, 2 \mathrm{H}, \mathrm{CH}_{2}$ ), 1.96 (quin, $J$ 7.3 Hz, $2 \mathrm{H}, \mathrm{CH}_{2}$ ), 1.28 (t, J 7.0 Hz, 3H, $\mathrm{CH}_{3}$ ); ${ }^{13} \mathrm{C} \mathrm{NMR} \mathrm{(75} \mathrm{MHz,} \mathrm{CDCl}_{3}$ ) $\delta 168.3,167.9,155.8,138.8$, 132.7, 130.6, 127.8, 126.9, 118.0, 100.6, 59.3, 51.1, 31.6, 21.8, 14.2; MS (EI) m/z 294 (8), 292 
(M+45, 8\%), 258 (45), 214 (16), 212 (13), 168 (65), 165 (98), 183 (100). Anal. Calcd for $\mathrm{C}_{15} \mathrm{H}_{16} \mathrm{NO}_{3} \mathrm{Br}$ : C, 53.27; H, 4.77; N, 4.14. Found: C, 53.26; H, 4.75; N, 3.97.

B. Synthesis of fused quinolin-4-one derivatives 9 from 8. Ethyl 5-oxo-1,2,3,5tetrahydropyrrolo[1,2-a]quinoline-4-carboxylate (9a). To a solution of 8a' in THF was added sodium hydride 9 ( 1 eq.), and the mixture was heated at $50{ }^{\circ} \mathrm{C}$ for $6 \mathrm{~h}$. After removal of the solvent, the residue was subjected to flash chromatography to afford 9a and 1a in $40 \%$ and $12 \%$ yield, respectively. 9a: colorless crystals; mp $164-5{ }^{\circ} \mathrm{C}$ (lit. ${ }^{26} 140-2{ }^{\circ} \mathrm{C}$ ); IR (KBr) 1692, 1653 $\mathrm{cm}^{-1} ;{ }^{1} \mathrm{H}$ NMR (300 MHz, $\left.\mathrm{CDCl}_{3}\right) \delta 8.46$ (d, $\left.J 8.4 \mathrm{~Hz}, 1 \mathrm{H}, \mathrm{ArH}\right), 8.43(\mathrm{~d}, J 7.9 \mathrm{~Hz}, 1 \mathrm{H}, \mathrm{ArH})$, 7.47 (t, $J 7.5 \mathrm{~Hz}, 1 \mathrm{H}, \mathrm{ArH}), 6.69$ (t, $J 7.9 \mathrm{~Hz}, 1 \mathrm{H}, \mathrm{ArH}), 4.42$ (q, $\left.J 6.9 \mathrm{~Hz}, 2 \mathrm{H}, \mathrm{OCH}_{2}\right), 4.24$ (t, $J$ $7.4 \mathrm{~Hz}, 2 \mathrm{H}, \mathrm{CH}_{2}$ ), 3.50 (t, $J 7.8 \mathrm{~Hz}, 2 \mathrm{H}, \mathrm{CH}_{2}$ ), 2.22 (quin, $J 7.5 \mathrm{~Hz}, 2 \mathrm{H}, \mathrm{CH}_{2}$ ), 1.44 (t, $J 6.9 \mathrm{~Hz}$, $\left.3 \mathrm{H}, \mathrm{CH}_{3}\right) ;{ }^{13} \mathrm{C}$ NMR $\left(75 \mathrm{MHz}, \mathrm{CDCl}_{3}\right) \delta 166.4,161.2,150.9,135.3,132.7,127.3,126.2,124.9$, 124.5, 104.2, 60.8, 48.6, 34.0, 21.2, 14.4; MS (EI) m/z 258 (18), 257 ( $\left.{ }^{+}, 100 \%\right), 229$ (24), 228 (22), 212 (87), 210 (40). Anal. Calcd for $\mathrm{C}_{15} \mathrm{H}_{15} \mathrm{NO}_{3}$ : C, 70.02; H, 5.88; N, 5.44. Found: C, 70.05; H, 5.86; N, 5.30 .

Ethyl 6-oxo-2,3,4,6-tetrahydro-1H-pyrido[1,2-a]quinoline-5-carboxylate (9b). The intermediate $\mathbf{8 b}$, obtained following the same procedure as for $\mathbf{8 a}$, was not purified by column chromatography. Following the cyclization reaction as for $\mathbf{9 a}$, $\mathbf{9 b}$ was obtained in $52 \%$ yield based on starting heterocyclic enamine $\mathbf{1 b}$. 9b: colorless crystals; mp $133-4{ }^{\circ} \mathrm{C}$ (lit. ${ }^{26} 132-5{ }^{\circ} \mathrm{C}$ ); IR (KBr) 1716, $16191600 \mathrm{~cm}^{-1}$; ${ }^{1} \mathrm{H}$ NMR (300 MHz, $\mathrm{CDCl}_{3}$ ) $\delta 8.47$ (d, J 7.9 Hz, 1H, ArH), 7.65 (t, $J 7.3 \mathrm{~Hz}, 1 \mathrm{H}, \mathrm{ArH}), 7.54$ (d, $J 8.7 \mathrm{~Hz}, 1 \mathrm{H}, \mathrm{ArH}), 7.39$ (d, $J 7.5 \mathrm{~Hz}, 1 \mathrm{H}, \operatorname{ArH}), 4.41$ (q, J 7.1 $\mathrm{Hz}, 2 \mathrm{H}, \mathrm{OCH}_{2}$ ), 4.12 (t, $J 6.3 \mathrm{~Hz}, 2 \mathrm{H}, \mathrm{CH}_{2}$ ), 3.04 (t, $J 6.5 \mathrm{~Hz}, 2 \mathrm{H}, \mathrm{CH}_{2}$ ), 2.12 (quin, $6.1 \mathrm{~Hz}, 2 \mathrm{H}$, $\left.\mathrm{CH}_{2}\right), 1.93-1.85\left(\mathrm{~m}, 2 \mathrm{H}, \mathrm{CH}_{2}\right), 1.40\left(\mathrm{t}, J 7.1 \mathrm{~Hz}, 3 \mathrm{H}, \mathrm{CH}_{3}\right) ;{ }^{13} \mathrm{C}$ NMR $\left(75 \mathrm{MHz}, \mathrm{CDCl}_{3}\right) \delta 173.4$, 167.5, 149.9, 140.8, 132.2, 126.7, 126.6, 124.0, 116.7, 114.7, 61.2, 46.4, 27.6, 22.5, 18.3, 14.2; MS (EI) m/z 272 (5), 271 (M+2, 24\%), 226 (47), 199 (100). Anal. Calcd for $\mathrm{C}_{16} \mathrm{H}_{17} \mathrm{NO}_{3}$ : C, 70.83; H, 6.32; N, 5.16. Found: C, 70.64; H, 6.33; N, 5.04.

Ethyl 5-oxo-5,7,8,9,10,11-hexahydroazapino[1,2-a]quinoline-6-carboxylate (9c). Similar to $\mathbf{9 b}$, the reaction of 1c with o-cholorobenzoyl chloride, followed by the NaH-promoted cyclization gave 9c in 39\% yield (based on 1c). 9c: colorless crystals; mp 153-4 ${ }^{\circ} \mathrm{C}$ (lit. ${ }^{26} 146-8$ $\left.{ }^{\circ} \mathrm{C}\right)$; IR (KBr) 1735, 1616, $1595 \mathrm{~cm}^{-1} ;{ }^{1} \mathrm{H}$ NMR $\left(300 \mathrm{MHz}, \mathrm{CDCl}_{3}\right) \delta 8.41(\mathrm{~d}, J 8.1 \mathrm{~Hz}, 1 \mathrm{H}$, ArH), 7.60 (t, $J 7.5 \mathrm{~Hz}, 7.46$ (d, J $8.7 \mathrm{~Hz}, 1 \mathrm{H}, \mathrm{ArH}$ ), 7.31 (t, $J .5 \mathrm{~Hz}, 1 \mathrm{H}, \mathrm{ArH}), 4.38$ (q, J 7.0 $\left.\mathrm{Hz}, 2 \mathrm{H}, \mathrm{OCH}_{2}\right), 4.30-4.27\left(\mathrm{~m}, 2 \mathrm{H}, \mathrm{CH}_{2}\right), 2.96-2.93\left(\mathrm{~m}, 2 \mathrm{H}, \mathrm{CH}_{2}\right), 1.84-1.81(\mathrm{~m}, 6 \mathrm{H}$, $\mathrm{CH}_{2} \mathrm{CH}_{2} \mathrm{CH}_{2}$ ), 1.37 (t, $\left.J 6.2 \mathrm{~Hz}, 3 \mathrm{H}, \mathrm{CH}_{3}\right) ;{ }^{13} \mathrm{C} \mathrm{NMR}\left(75 \mathrm{MHz}, \mathrm{CDCl}_{3}\right) \delta 174.0,167.5,154.2$, 140.3, 132.2, 126.4, 126.0, 123.2, 116.9, 115.0, 61.0, 46.9, 31.5, 27.5, 26.4, 25.4, 14.1; MS (EI) m/z 286 (4), 285 ( $\left.\mathrm{M}^{+}, 22 \%\right), 240$ (40), 213 (100). Anal. Calcd for $\mathrm{C}_{17} \mathrm{H}_{19} \mathrm{NO}_{3}$ : C, 71.56; H, 6.71; N, 4.91. Found: C, 71.23; H, 6.70; N, 4.70.

\section{Acknowledgements}

We are grateful to the Major State Basic Research Development Program of China (Grant No. G2000077502) and the National Natural Science Foundation of China for financial support. 


\section{References}

1. Bertele, E.; Boos, H.; Dinitz, J. D.; Elsinger, F.; Eschenmoser, A.; Felner, I.; Gribi, H. P.; Gshwend, H.; Meyer, E. F.; Pesaro, M.; Scheffold, R. Angew. Chem., Int. Ed. Engl. 1964, 3, 490.

2. Taguchi, H.; Yazawa, H.; Arnett, J. F.; Kishi, Y. Tetrahedron Lett. 1977, 627.

3. Danishefsky, S.; Etheredge, S. J. J. Org. Chem. 1974, 39, 3430.

4. Shen, W.; Coburn, C. A.; Bornmann, W. G.; Danishefsky, S. J. Org. Chem. 1993, 58, 611.

5. Snyder, L.; Shen, W.; Bornmann, W. G.; Danishefsky, S. J. Org. Chem. 1994, 59, 7033.

6. Luly, J. R.; Rapoport, H. J. Am. Chem. Soc. 1983, 105, 2859 and references cited therein.

7. Pinnick, H. W.; Chang, Y.-H. J. Org. Chem. 1978, 43, 4662.

8. Buchanan, J. G.; Jigajinni, V. B.; Singh, G.; Wightman, R. T. J. Chem. Soc. Perkin Trans. 1 1987, 2377.

9. Paulvannan, K.; Stille, J. R. J. Org. Chem. 1994, 59, 1613.

10. Saliou, C.; Fleurant, A.; Celerier, J. P.; Lhommet, G. Tetrahedron Lett. 1991, 32, 3365.

11. Fleurant, A.; Celerier, J. P.; Lhommet, G. Tetrahedron: Asymmetry 1993, 4, 1429.

12. Thanh, G. V.; Celerier, J. P.; Lhommet, G. Tetrahedron: Asymmetry 1996, 7, 2211.

13. Folmer, J. J.; Acero, C.; Thai, D. L.; Rapoport, H. J. Org. Chem. 1998, 63, 8170.

14. Brunerie, P.; Celerier, J. P.; Petit, H.; Lhommet, G. J. Heterocycl. Chem. 1986, 23, 1183.

15. Bahaji, El. H.; Refouvelet, B.; Couquelet, J.; Tronche, P. C. R. Acad. Sc. II 1986, 303, 455.

16. Nagasaka, T.; Inoue, H.; Ichimura, M.; Hamaguchi, F. Synthesis 1982, 848.

17. Brunerie, P.; Celerier, J. P.; Huche, M.; Lhommet, G. Synthesis 1985, 735.

18. Wang, M.-X.; Miao, W.-S.; Cheng, Y.; Huang, Z.-T. Tetrahedron 1999, 55, 14611.

19. Cheng, Y.; Yang, H.-B.; Wang, M.-X.; Williams, D. J. Tetrahedron 2002, 58, 2821.

20. For a useful review, see: Roeder, E. Curr. Org. Chem. 1999, 3, 557.

21. For the synthesis of hydroxylated indolizidine alkaloidal compounds, see: (a) Maggini, M.; Prato, M.; Ranelli, M.; Scorrano, G. Tetrahedron Lett. 1992, 33, 6537. (b) Cordero, F. M.; Cicchi, S.; Goti, A.; Brandi, A. Tetrahedron Lett. 1994, 35, 949. (c) Cicchi, S.; Goti, A.; Brandi, A. J. Org. Chem. 1995, 60, 4743.

22. Cheng, Y.; Zhao, M.; Wang, M.-X.; Wang, L.-B.; Huang, Z.-T. Synth. Commun. 1995, 25, 1339.

23. Wang, M.-X.; Wu, X.-D.; Wang, L.-B.; Huang, Z.-T. Synth. Commun. 1995, 25, 343. For reviews of the chemistry of heterocyclic ketene aminals see: (a) Huang, Z.-T.; Wang, M.-X. Heterocycles, 1994, 37, 1233. (b) Wang, M.-X.; Huang, Z.-T. Progress Nat. Sci. 2002, 12, 249.

24. Michael, J. P.; Chang, S. F.; Wilson, C. Tetrahedron Lett. 1993, 34, 8365.

25. (a) Mueller, L. G.; Lawton, R. G. J. Org. Chem. 1979, 44, 4742. (b) Franck, R. W.; Bernardy, K. F. J. Org. Chem. 1968, 33, 3050.

26. Coppola, G. M.; Damon, R. E. J. Heterocycl. Chem. 1980, 17, 1729. 